\title{
Fatigue, reduced sleep quality and restless legs syndrome in Charcot-Marie-Tooth disease: a web-based survey
}

\author{
Matthias Boentert $\cdot$ Rainer Dziewas $\cdot$ Anna Heidbreder $\cdot$ Svenja Happe $\cdot$ \\ Ilka Kleffner · Stefan Evers $\cdot$ Peter Young
}

Received: 28 July 2009/Revised: 7 September 2009/Accepted: 9 November 2009/Published online: 24 November 2009

(C) Springer-Verlag 2009

\begin{abstract}
To investigate the prevalence of fatigue, daytime sleepiness, reduced sleep quality, and restless legs syndrome (RLS) in a large cohort of patients with CharcotMarie-Tooth disease (CMT) and their impact on healthrelated quality of life (HRQoL). Participants of a web-based survey answered the Epworth Sleepiness Scale, the Pittsburgh Sleep Quality Index, the Multidimensional Fatigue Inventory, and, if the diagnostic criteria of RLS were met, the International RLS Severity Scale. Diagnosis of RLS was affirmed in screen-positive patients by means of a standardized telephone interview. HRQoL was assessed by using the SF-36 questionnaire. Age- and sex-matched control subjects were recruited from waiting relatives of surgical outpatients. 227 adult self-reported CMT patients answered the above questionnaires, $42.9 \%$ were male, and $57.1 \%$ were female. Age ranged from 18 to 78 years. Compared to controls $(n=234)$, CMT patients reported significantly higher fatigue, a higher extent and prevalence of daytime sleepiness and worse sleep quality. Prevalence of RLS was $18.1 \%$ in CMT patients and 5.6\% in controls
\end{abstract}

Electronic supplementary material The online version of this article (doi:10.1007/s00415-009-5390-1) contains supplementary material, which is available to authorized users.

M. Boentert · R. Dziewas · A. Heidbreder · I. Kleffner ·

S. Evers · P. Young $(\square)$

Department of Neurology, University Hospital Münster,

Albert-Schweitzer-Str. 33, 48129 Münster, Germany

e-mail: young@uni-muenster.de

M. Boentert

e-mail: mboenter@uni-muenster.de

S. Happe

Department of Clinical Neurophysiology,

Klinikum Bremen-Ost/University of Göttingen,

Züricher Strasse 40, 28325 Bremen, Germany $(p=0.001)$. RLS severity was correlated with worse sleep quality and reduced HRQoL. Women with CMT were affected more often and more severely by RLS than male patients. With regard to fatigue, sleep quality, daytime sleepiness, RLS prevalence, RLS severity, and HRQoL, we did not find significant differences between genetically distinct subtypes of CMT. HRQoL is reduced in CMT patients which may be due to fatigue, sleep-related symptoms, and RLS in particular. Since causative treatment for CMT is not available, sleep-related symptoms should be recognized and treated in order to improve quality of life.

Keywords Charcot-Marie-Tooth disease $\cdot$ Fatigue . Restless legs syndrome $\cdot$ Quality of life

\section{Introduction}

Charcot-Marie-Tooth disease (CMT) refers to a heterogenous group of monogenetic peripheral neuropathies with an overall prevalence of 1:2,500 [1]. Classification of CMT is based on clinical and electrophysiological findings, the trait of inheritance, and the underlying genetic cause. To date, 40 genes and gene loci have been associated with CMT [2]. Distal motor weakness and muscle atrophy, foot deformities, gait disturbances and sensory impairment are the clinical hallmarks of CMT. For self-reporting of CMT subtypes the following options were offered: CMT1, CMT2, CMTX and "other". Many CMT patients report fatigue, daytime sleepiness, and non-restorative sleep $[3,4]$. Sleep disturbances may be associated with paresthesia, muscle cramps, or restless legs syndrome (RLS). In a study with 44 participants, RLS was present in $37 \%$ of CMT2 patients and $0 \%$ of CMT1 patients [5]. Recently, RLS was found to be reported by $16.1 \%$ of CMT2 and 
$10.0 \%$ of CMT1 patients [6]. Disordered breathing during sleep in CMT may be associated with phrenic neuropathy and diaphragmatic weakness [7, 8]. Sleep apnea was found to be common in CMT patients, and the apnea-hypopneaindex (AHI) correlated with disease severity [9, 10]. The objective of the present study was to determine the prevalence of fatigue, daytime sleepiness, reduced sleep quality and RLS in CMT patients and their impact on healthrelated quality of life (HRQoL).

\section{Methods}

Data were collected by means of an anonymized, webbased survey which was made accessible exclusively to persons registered with the only German internet forum for CMT patients [11]. Registered subjects were invited to participate by the responsible webmaster via e-mail. It was made impossible to take part more than once. The study was approved by the local ethics committee. Participants were asked whether the diagnosis of CMT had been made by a specialized neurologist, and whether it had been genetically confirmed. For self-reporting of CMT subtypes the following options were offered: "CMT1 (PMP22 or MPZ gene)", "CMT2 (Mitofusin-2 or other genes)", "CMTX (GJB1 gene)", and "other/unclassified CMT", the latter including participants without genetically proven diagnoses. Participants were asked for age, gender, height, and weight. In addition, they answered six questionnaires in the German language. The Epworth Sleepiness Scale (ESS) is an eight-item self-reporting tool to measure daytime sleepiness [12], with a score ranging from 0 to 24 . A cut-off score $>10$ is considered pathological [13]. The Pittsburgh Sleep Quality Index (PSQI) focusses on the quality of sleep during the last 4 weeks [14]. It comprises seven component scores reflecting sleep latency, sleep duration, habitual sleep efficiency, sleep disturbances, use of sleeping medication, daytime dysfunction, and overall sleep quality. The global PSQI score has a range of 0-21. A cut-off score of 5 is considered to allow reliable distinction between "good sleepers" $(\leq 5)$ and "bad sleepers" $(>5$, [15]). Fatigue was assessed using the Multidimensional Fatigue Inventory (MFI-20) which covers five dimensions of fatigue: general, physical, and mental fatigue, reduced activities, and reduced motivation [16]. The German version is based on a 5-point scale, resulting in a global score of 20-100, with a higher score indicating more severe fatigue [17]. RLS is a clinical diagnosis [18]. Participants answered standardized questions covering the diagnostic criteria of RLS as defined by the International Restless Legs Syndrome Study Group (German translation validated by [19]). In addition, we asked for any current medication and concomitant diseases possibly promoting RLS, fatigue, or breathing disorders during sleep. The respective list included known obstructive sleep apnea (OSA), chronic obstructive pulmonary disease, chronic renal failure, congestive heart failure, multiple sclerosis, iron deficiency, malignancy, and diabetes mellitus. Participants meeting the diagnostic criteria for RLS were contacted by e-mail, and then received a telephone call for confirmatory diagnostic evaluation by a movement disorder specialist (M. B.). The structured telephone interview was performed using the same RLS questionnaire, clarifying whether each diagnostic symptom was truly present. The interview was designed in accordance with a previously validated English diagnostic telephone interview for RLS [20]. Patients screen-positive for RLS were asked for any specific medication and answered the International RLS Severity Scale (IRLS, [21, 22]) which allows discrimination of four RLS severity grades: mild (score 0-10), moderate (score 11-20), severe (score 21-30), and very severe (score 31-40). The SF-36 questionnaire [23] yields scores for eight domains of HRQoL: physical functioning, physical role limitation, physical pain, general health perception, vitality, social functioning, emotional role limitation, and mental health perception. Physical and mental component summary scales (PCS and MCS) are calculated from the subscales and transformed to a normalized T-score with a mean of 50 and standard deviation of 10 [23]. Higher scores represent better quality of life. German versions of the ESS, the PSQI, the MFI-20 and the SF-36 have been validated [2429]. With the exception of the MFI-20 and the SF-36, all questionnaires of the survey were also answered by 234 age- and sex-matched control subjects who were recruited from waiting relatives of surgical patients visiting a traumatology outpatient clinic. As a random sample, this population was drawn from 411 controls we previously published [30].

\section{Statistical analysis}

The survey was conducted using GrafStat ${ }^{\circledR}$ software [31]. Data analysis was performed using SPSS $^{\circledR}$ v.15.0. Normal distribution of continuous variables was tested using the Kolmogorov-Smirnov test. Student's $t$ test and the Mann-Whitney $U$ test were used to compare the means of parametric or non-parametric variables, respectively. Differences among categorical variables were analysed using the $\chi^{2}$ test or the two-tailed Fisher's exact test as appropriate. For differences between groups, pairwise ANOVA with Bonferroni correction was applied. Spearman correlation coefficients were used to examine associations between continuous variables. $p$ Values $<0.05$ were considered statistically significant. Multiple linear regression analysis was performed to test whether age and sex were 
independently associated with MCS and PCS scores. Multivariate analysis was carried out to examine the association between the IRLS score and PCS or MCS scores while controlling for age, sex and the MFI-20, ESS, and PSQI scores. In order to limit the number of regressions, the MCS and PCS scores, rather than the individual SF-36 domains, were used as main outcome variables.

\section{Results}

247 adult survey participants reported having been diagnosed with CMT by a specialized neurologist. 20 of them incompletely answered at least one of the questionnaires and were excluded from further analysis. Of the remaining 227 individuals, $42.9 \%$ were male, and $57.1 \%$ were female. Patients were 18 to 78 years old (mean 42.1 years, SD 11.6 years). The mean body mass index (BMI) was 23.7 (SD 6.8). Both age and BMI were normally distributed. Representation of self-reported CMT subtypes in the study population was as follows: CMT1 49.3\%, CMT2 15.9\%, CMTX 4.0\%, "other and unclassified CMT" 30.8\%. Concomitant diseases were specified by 21 (9.3\%) of CMT patients. In the control group, mean age was 42.0 years (SD 12.9). 64.5\% of control subjects were female, and $35.5 \%$ were male.

\section{Fatigue}

Mean and standard deviations of the MFI-20 total score and subscores are depicted in Table 1. Genetically distinct CMT subgroups did not differ significantly (Table 2). In CMT patients, we found a weak but significant correlation between age and the General Fatigue subscore (GFS, $r=0.13, p<0.05)$. Neither age nor sex correlated with the MFI-20 total score or any of the other subscores. Since the GFS may serve as an overall indicator of fatigue [16], a cut-off value of 12 is considered to indicate severe fatigue in cancer patients [32]. The GFS was 12 or above in 98 (43.2\%) of the study's CMT patients. Patients with concomitant disease were analyzed separately. Mean MFI-20 total score and subscores were not significantly increased. However, the percentage of severely fatigued patients in this subgroup was $73.6 \%$. Compared to data obtained from the literature, the MFI-20 global score and the different subscores each were higher in self-reported CMT patients than in a cohort of healthy elderly people ( $>60$ years) drawn from the normal population (Table 1, [17]).

\section{Daytime sleepiness}

Mean ESS score was 8.8 (SD 4.1) in CMT patients and 6.5 (SD 3.2) in control subjects $(p<0.001)$. Pathological daytime sleepiness as indicated by an ESS score $>10$ was reported by $31.7 \%$ of CMT patients and $11.8 \%$ of nonCMT controls $(p<0.001)$. Differences between genetically distinct subgroups were not significant (Table 2). Age and sex were not correlated with the ESS total score. Multiple regression analysis showed that the presence of CMT was not a significant predictor of excessive daytime sleepiness (data not shown).

\section{Reduced sleep quality}

$79.3 \%$ of CMT patients and $17.2 \%$ of controls turned out to be "bad sleepers" as indicated by a global PSQI above 5 $(p<0.001)$. A PSQI global score $>5$ was found with a prevalence of $80.4 \%$ in the CMT1 group, $72.2 \%$ in the CMT2 group, $88.9 \%$ in the CMTX group, and $80.0 \%$ in the "other/unclassified CMT" subgroup. Mean PSQI global

Table 1 Self-reported fatigue in CMT patients

\begin{tabular}{llllll}
\hline Study population & Present study & & & \\
\cline { 2 - 6 } & $\begin{array}{l}\text { CMT1, CMT2, CMTX } \\
\text { (with known genetic diagnosis) }\end{array}$ & $\begin{array}{l}\text { Other/unclassified } \\
\text { CMT }\end{array}$ & $\begin{array}{l}\text { All CMT } \\
\text { patients }\end{array}$ & $\begin{array}{l}\text { CMT and concomitant } \\
\text { disease }\end{array}$ & $\begin{array}{l}\text { Elderly } \\
n\end{array}$ \\
\hline Age (mean and SD) & 157 & 70 & 227 & 21 & 622 \\
MFI-20 global score & $60.2(5.1)$ & $42.2(12.5)$ & $41.2(12.6)$ & $50.4(8.6)$ & $69.6(6.8)$ \\
General fatigue & $11.1(2.0)$ & $59.5(4.6)$ & $60.2(4.9)$ & $60.6(3.9)$ & $50.6(16.9)$ \\
Physical fatigue & $12.9(1.8)$ & $11.1(1.7)$ & $11.2(1.9)$ & $11.8(2.2)$ & $10.6(3.8)$ \\
Mental fatigue & $11.4(1.8)$ & $12.6(1.6)$ & $12.8(1.8)$ & $12.3(1.9)$ & $10.8(4.3)$ \\
Reduced activities & $12.7(1.7)$ & $11.5(1.7)$ & $11.4(1.8)$ & $11.8(1.9)$ & $9.0(3.5)$ \\
Reduced motivation & $12.0(2.3)$ & $12.2(1.7)$ & $12.6(1.7)$ & $12.5(2.0)$ & $10.5(4.1)$ \\
\hline
\end{tabular}

Self-reported fatigue in CMT patients. Numbers depict mean and standard deviation (SD) of the MFI-20 global and domain scores. Patients with CMT and concomitant disease reached even higher scores. For comparison, the far right column (1) shows data obtained from 622 elderly people (>60 years) in the German general population [17]. Data obtained from patients who identified themselves as genetically diagnosed with CMT1, CMT2 or CMTX were combined since ANOVA analysis did not show any differences between these subgroups (Table 2) 
Table 2 Intergroup analyses among genetically distinct CMT subgroups

\begin{tabular}{|c|c|c|c|c|}
\hline & CMT1 & СMT2 & CMTX & $p$ value \\
\hline$n$ & 112 & 36 & 9 & - \\
\hline Age & $42.2(11.1)$ & $41.6(12.0)$ & $43.9(9.9)$ & $0.81 *$ \\
\hline Females $(\%)$ & 83.3 & 57.1 & 0 & $0.29^{\#}$ \\
\hline Fatigue (MFI-20 global score) & $60.9(5.1)$ & $59.4(4.2)$ & $58.2(6.3)$ & $0.10 *$ \\
\hline Daytime sleepiness (ESS score) & $9.2(4.1)$ & $8.6(4.0)$ & $6.2(4.2)$ & $0.10 *$ \\
\hline $\mathrm{ESS}>10(\%)$ & 38.4 & 30.6 & 33.3 & $0.73^{\#}$ \\
\hline Reduced sleep quality (PSQI global score) & $8.1(2.9)$ & $7.6(2.8)$ & $7.8(2.4)$ & $0.84 *$ \\
\hline PSQI > $5(\%)$ & 80.4 & 72.2 & 88.9 & $0.68^{\#}$ \\
\hline RLS prevalence $(\%)$ & 22.3 & 19.4 & 11.1 & $0.83^{\#}$ \\
\hline RLS severity (IRLS score) & $20.7(6.2)$ & $21.2(4.9)$ & 16.0 & $0.10^{*}$ \\
\hline
\end{tabular}

Differences between the CMT1, CMT2, and CMTX subgroups with regard to all outcomes of interest. Numbers are depicted as mean and SD or percentage as indicated. $p$ Values $<0.05$ were considered significant. Statistical analysis was carried out using ANOVA with Bonferroni's correction (*) or Pearson's Chi-square test (\#), respectively. In the CMTX group, only one patient had RLS

Table 3 PSQI global and component scores of self-reported CMT patients and control subjects

\begin{tabular}{|c|c|c|c|c|c|c|}
\hline & \multicolumn{5}{|l|}{ Present study } & \multirow[t]{2}{*}{ (1) } \\
\hline & $\begin{array}{l}\text { CMT1, CMT2, CMTX } \\
\text { (with known genetic diagnosis) }\end{array}$ & $\begin{array}{l}\text { Other/unclassified } \\
\text { CMT }\end{array}$ & $\begin{array}{l}\text { All CMT } \\
\text { patients }\end{array}$ & $\begin{array}{l}\text { CMT and } \\
\text { concomitant disease }\end{array}$ & Controls & \\
\hline$n$ & 157 & 70 & 227 & 21 & 234 & 1,049 \\
\hline Age & $41.5(11.0)$ & $42.2(12.5)$ & $41.2(12.6)$ & $50.4(8.6)$ & $42.0(12.9)$ & 41.9 \\
\hline PSQI global score & $8.0(2.8)$ & $7.8(2.8)$ & $7.9(2.8)$ & $8.7(3.4)$ & $3.7(2.3)$ & $4.6(3.7)$ \\
\hline Sleep quality & $1.4(0.7)$ & $1.4(0.7)$ & $1.4(0.7)$ & $1.6(0.9)$ & $0.7(0.6)$ & $0.8(0.8)$ \\
\hline Sleep latency & $1.6(0.9)$ & $1.4(0.9)$ & $1.5(0.9)$ & $1.6(1.0)$ & $0.6(0.7)$ & $0.9(0.9)$ \\
\hline Sleep duration & $1.0(0.7)$ & $0.8(0.7)$ & $0.9(0.7)$ & $1.3(1.1)$ & $0.6(0.7)$ & $0.7(0.7)$ \\
\hline Sleep efficiency & $0.9(1.0)$ & $0.9(1.1)$ & $0.9(1.0)$ & $1.1(1.2)$ & $0.3(0.7)$ & $0.3(0.7)$ \\
\hline Sleep disturbances & $1.5(0.6)$ & $1.4(0.6)$ & $1.5(0.6)$ & $1.7(0.7)$ & $0.9(0.4)$ & $0.8(1.0)$ \\
\hline Sleep medication & $0.1(0.3)$ & $0.2(0.6)$ & $0.1(0.4)$ & $0.0(0.0)$ & $1.3(0.1)$ & $0.2(0.7)$ \\
\hline Daytime dysfunction & $1.6(0.7)$ & $1.6(0.7)$ & $1.6(0.7)$ & $1.5(0.8)$ & $0.7(0.6)$ & $0.8(0.8)$ \\
\hline
\end{tabular}

PSQI global and component scores of self-reported CMT patients and control subjects. The far right column (1) shows data obtained from a large cohort of the Austrian normal population using the German version of the PSQI [28]. Numbers depict mean and standard deviation (in brackets). In CMT patients, PSQI global score and 6 out of the 7 component scores were significantly higher than in control subjects (numbers are printed in bold, $p<0.001$ ). Patients with CMT and concomitant disease reached even higher scores on the PSQI. Data obtained from patients who identified themselves as genetically diagnosed with CMT1, CMT2 or CMTX were combined since ANOVA analysis did not show any differences between these subgroups

score was 7.9 (SD 2.8) in all CMT patients and 3.7 (SD $2.3)$ in the control group $(p<0.001)$. Differences between genetically distinct subgroups were not significant (Table 2). Six out of seven component scores were significantly higher in CMT patients than in controls $(p<0.001$, Table 3). Age and sex did not correlate with the global PSQI or any of the component scores. Multiple regression analysis including both CMT and control subjects revealed CMT as the only significant predictor of reduced sleep quality independent from age and sex $(p<0.001)$.

\section{RLS and RLS severity}

$47(21.9 \%)$ self-reported CMT patients and $13(5.6 \%)$ control subjects were screen-positive for RLS $(p<0.001)$. Diagnosis of RLS was confirmed by personal interview in 41 of the screen-positive CMT patients. The resulting prevalence of RLS among all CMT patients was $18.1 \%$, which was still significantly higher than in the control group $(p<0.001)$. The prevalence of RLS was $22.3 \%$ in the CMT1 group, $19.4 \%$ in the CMT2 group, and $12.9 \%$ in the "other/unclassified CMT" subgroup (n. s., Table 2). 
Only one out of nine CMTX patients had RLS. Mean onset of RLS symptoms was at 27.7 years of age, with a range from 4 to 61 years. RLS was most common in participants above 60 years of age $(23.5 \%)$, compared to $21.3 \%$ in the 30-60 years group and $16.7 \%$ in patients under 30 years (n.s.). $24.6 \%$ of women and $15.5 \%$ of men had RLS $(p=0.09)$. Women made up the majority of CMT patients with RLS (68.1\%), and they reached significantly higher IRLS scores than men $(20.7$ vs. $16.0, p<0.05)$. In the CMT group, severity of RLS was not age-dependent (data not shown). 20 out of 41 CMT patients with RLS (48.8\%) specified that they had been taking anticonvulsants, antidepressants, or L-DOPA for more than 6 months. $12.2 \%$ reported receiving iron supplementation for proven iron deficiency. All RLS patients on medication reported a decrease of RLS symptoms after the onset of drug therapy. However, the mean IRLS score was only slightly lower in patients on symptomatic treatment than in patients without any specific medication (data not shown).

Intercorrelation of fatigue, reduced sleep quality, daytime sleepiness, and RLS

We did not find significant correlations between either the MFI-20 and ESS scores or the MFI-20 and PSQI scores in CMT patients (data not shown). Impaired sleep quality (PSQI) and daytime sleepiness (ESS) were not significantly correlated in the entire CMT group $(r=0.12, p=0.55)$, the non-RLS subgroup $(r=0.29, p=0.13)$, or in the subset of patients with both CMT and RLS ( $r=0.15$, $p=0.33)$. However, ESS and PSQI were significantly correlated $(r=0.51, p=0.02)$ in female CMT patients with RLS. Severity of RLS symptoms and global PSQI were significantly correlated $(r=0.43, p<0.05)$ in CMT patients with RLS. This correlation was based on the female patients alone $(r=0.54, p=0.02)$. The IRLS score showed no correlation with the MFI-20 global score or the ESS score, respectively.

Impact of fatigue and sleep-related symptoms on quality of life

Among self-reported CMT patients, the lowest SF-36 subscores were found for the vitality domain score (38.0, SD 19.4) and the physical component score (PCS, 34.4, SD 10.8). Bivariate analysis showed that global PSQI and ESS scores each were inversely correlated with four of the SF36 subscores (see Table 4, supplemental data). The MFI-20 total score was inversely correlated only with the physical functioning index. In patients with both CMT and RLS, we found a strong inverse correlation between RLS severity and the physical functioning index, the role-physical score, and the PCS ( $r$ values $-0.56,-0.64,-0.66, p$ values
$<0.01)$. Inverse correlation between RLS severity and the mental health perception, vitality, and social functioning indices was also significant ( $r$ values $-0.46,-0.42$, and $-0.43, p$ values $<0.05)$. Multivariate analysis was performed separately for CMT patients with and without RLS. We did not find any significant predictors for SF-36 MCS in both groups. In patients with RLS, the significant independent predictors of SF-36 PCS were age $(\beta=-0.33$, $p<0.01)$, ESS score $(\beta=-0.19, p<0.05)$, and the IRLS score $(\beta=-0.66, p<0.01)$. In this subgroup, the IRLS score remained a significant independent predictor of the PCS after controlling for age, sex, PSQI, ESS, and MFI-20 scores $(\beta=-0.5, p<0.05)$. In CMT patients without RLS, only age was a significant predictor of the PCS $(\beta=-0.43, p<0.01)$.

\section{Discussion}

We present here the results of a survey on fatigue, sleep quality, RLS, and HRQoL in a large cohort of self-reported CMT patients. Prior studies focussed on either fatigue [3, 33], RLS [5, 6] or daytime sleepiness [4] in smaller numbers of patients. Using the Checklist Individual Strength (CIS), Kalkman et al. [3] reported severe fatigue in $64 \%$ of 137 CMT1A patients. Based on the GFS of the MFI-20, we found severe fatigue in $43.2 \%$ of survey participants. The use of different scoring tools may explain this variation. In accordance with the study mentioned, we did not find a correlation between age and the MFI-20 global score. Compared to previous studies on fatigue in the general population, CMT patients of all subgroups showed higher MFI-20 subscores than people above 60 years of age [17]. Whereas age correlates with fatigue in the general population [29], it is apparently not a major factor contributing to fatigue in CMT patients. However, the GFS was significantly correlated with age, which possibly reflects that the progressive course of CMT takes its toll in older patients. Little is known about the mechanisms by which peripheral neuropathy leads to fatigue. It might be explained by walking difficulties due to motor weakness, foot deformities, and loss of proprioceptive control. However, some patients report fatigue in the presence of rather mild neurological disability. Our results suggest that in CMT, fatigue is not correlated with poor sleep or daytime sleepiness. It has been postulated that fatigue and daytime sleepiness are different entities both caused by non-restorative sleep [13], and our findings are in accordance with previous reports showing that in multiple sclerosis, for example, only a subset of ESS items is correlated with fatigue [34].

The prevalence of impaired sleep and excessive daytime sleepiness is extraordinarily high in self-reported CMT 
patients, with no significant differences between genetic subtypes. Thus, our study cannot verify the assumption that sleep quality is worse in CMT2 patients possibly due the higher prevalence of RLS in this group previously reported by Gemignani et al. [5]. Difficulties in falling asleep and frequent awakenings appear to be the most important reasons for non-restorative sleep in CMT patients. Our data suggest that many of them suffer from RLS. We found the overall prevalence of RLS in CMT patients to be $18.1 \%$, which is similar to numbers recently reported by Hattan et al. [6]. Whereas Hattan et al. [6] found the highest proportion of RLS-positive patients in individuals with sensory and autonomous neuropathy (HSAN), this subgroup cannot be identified in our study population. In contrast to previous studies [5, 6] we found patients with CMT1 slightly more often affected by RLS than CMT2 patients, and the genetic subtype did not significantly influence RLS severity. These findings may in part be explained by two reasons: First, in our study, $83.3 \%$ of CMT1 patients and only $57.1 \%$ of CMT2 patients were female, and prevalence of RLS was about $10 \%$ higher in women than in men. Second, albeit primary axonal damage probably predisposes to RLS more than demyelination [35], patients with demyelinating neuropathy do experience secondary axonal degeneration, and there is a close relationship between axonal loss and disability [36]. Among 20 CMT1 patients, Hattan et al. [6] reported a $10 \%$ prevalence of RLS, which is only half of what we found in 112 individuals. Since Hattan et al. did not provide epidemiological data on the CMT patients included in their study, it may be assumed that variations in age and sex distribution partially account for this difference. For reasons to be clarified, female CMT patients were affected by RLS more often and more severely than men, and the impact of RLS on sleep quality was higher in women.

Daytime sleepiness in CMT has been addressed by two studies using CMT1A patients as a control group for patients with myotonic dystrophy (MD) [4, 33]. Rubinsztein et al. [33] reported CMT1A patients to be less affected by daytime sleepiness than MD patients, and none of them scored $>10$ on the ESS. Phillips et al. [4] found 5 out of 13 CMT patients reached an ESS score $>10$, and mean ESS score was 10.5. In our study population, with mean age lower and probably less affected persons included, the mean ESS score was only 8.8, but significantly higher than in the control group. Interestingly, ESS and global PSQI score were correlated only in (female) CMT patients with RLS, which may be explained by two circumstances: First, the PSQI reflects sleep quality rather than restorativeness of sleep. Second, poor sleep and excessive daytime sleepiness may go together only in the presence of a condition which massively reduces sleep quality. In many CMT patients, this condition is likely to be RLS. A substantial number of patients with RLS show periodic limb movements during sleep (PLMS) on polysomnography (PSG), possibly adding to sleep impairment [37, 38]. Gemignani et al. [5] reported that two-thirds of the CMT2 patients with RLS they studied had PLMS. Additional PSG studies are needed to further elucidate the impact of PLMS in CMT.

Disordered breathing during sleep may also be responsible for non-restorative sleep in CMT patients [39]. Phrenic neuropathy and nocturnal hypoventilation have been described in severely affected individuals [7, 8]. We and others reported sleep apnea in a high percentage of patients with CMT1A [9, 10]. Most patients mainly had obstructive apneas, probably caused by pharyngeal neuropathy and increased upper airway compliance. Future PSG studies should address this issue by evaluating pharyngeal function. Sleep apnea syndrome is a prominent cause of excessive daytime sleepiness. Since in our study ESS scores did not differ significantly between the CMT1 and CMT2 subgroups, it will be especially important to investigate the contribution of obstructive sleep apnea to daytime sleepiness in CMT2 patients.

It is a limitation of our study that objective clinical data on neurological impairment were not available. This fact is inherent to the survey approach we chose, and it makes it impossible to support the assumption that sleeprelated symptoms are correlated with disease severity in CMT, as we showed before in a small group of CMT1A patients with OSA [10]. For this purpose, standardized scoring tools such as the CMT neuropathy scale should be applied in future studies [40]. For the benefit of a large study population, we put up with the fact that the genetic diagnosis of participants could not be verified. The unexpected small number of individuals with CMTX indicates that self-reported data on genetic diagnoses have to be interpreted carefully, making it difficult to ascertain differences between CMT subgroups. However, the percentage of participants who reported having CMT1 or CMT2 appears to be in accordance with the estimated prevalence of the most common CMT subtypes [1]. Keeping these aspects in mind, it is the quintessence of our survey that HRQoL is markedly reduced in CMT patients, which is in keeping with previous reports [41, 42]. This may be due to excessive daytime sleepiness, substantial fatigue, poor sleep, and the presence of RLS in particular. Since causative treatment for CMT is not available, therapy should not be restricted to physiotherapy, walking aids and proper foot care. Patients should be asked for sleep-related symptoms and fatigue in order to identify treatable conditions. Apnea screening is appropriate in patients with at least moderate disability, and PSG should initially be performed if severe RLS, PLMS or nocturnal hypoventilation are suspected. 
Acknowledgment The authors are grateful to Stefan Laube and Jens Sommer, both Department of Neurology, University Hospital Münster, for their help in planning the web-based survey. We wish to thank Dr. Ellen M.A. Smets, Department of Medical Psychology, University of Amsterdam, for providing permission to use the Multidimensional Fatigue Inventory.

\section{References}

1. Kuhlenbaumer G, Young P, Hunermund G et al (2002) Clinical features and molecular genetics of hereditary peripheral neuropathies. J Neurol 249(12):1629-1650

2. http://www.molgen.ua.ac.be/CMTMutations

3. Kalkman JS, Schillings ML, van der Werf SP et al (2005) Experienced fatigue in facioscapulohumeral dystrophy, myotonic dystrophy, and HMSN-I. J Neurol Neurosurg Psychiatry 76(10):1406-1409

4. Phillips MF, Steer HM, Soldan JR et al (1999) Daytime somnolence in myotonic dystrophy. J Neurol 246(4):275-282

5. Gemignani F, Marbini A, Di Giovanni G et al (1999) CharcotMarie-Tooth disease type 2 with restless legs syndrome. Neurology 52(5):1064-1066

6. Hattan E, Chalk C, Postuma RB (2009) Is there a higher risk of restless legs syndrome in peripheral neuropathy? Neurology 72(11):955-960

7. Chan CK, Mohsenin V, Loke J et al (1987) Diaphragmatic dysfunction in siblings with hereditary motor and sensory neuropathy (Charcot-Marie-Tooth disease). Chest 91(4):567-570

8. Osanai S, Akiba Y, Nakano H et al (1992) Charcot-Marie-Tooth disease with diaphragmatic weakness. Intern Med 31(11):1267-1270

9. Dematteis M, Pepin JL, Jeanmart M et al (2001) Charcot-MarieTooth disease and sleep apnoea syndrome: a family study. Lancet 357(9252):267-272

10. Dziewas R, Waldmann N, Bontert M et al (2008) Increased prevalence of obstructive sleep apnoea in patients with CharcotMarie-Tooth disease: a case control study. J Neurol Neurosurg Psychiatry 79(7):829-831

11. http://www.hmsn.de

12. Johns MW (1991) A new method for measuring daytime sleepiness: the Epworth sleepiness scale. Sleep 14(6):540-545

13. Hossain JL, Ahmad P, Reinish LW et al (2005) Subjective fatigue and subjective sleepiness: two independent consequences of sleep disorders? J Sleep Res 14(3):245-253

14. Buysse DJ, Reynolds CF III, Monk TH et al (1989) The Pittsburgh Sleep Quality Index: a new instrument for psychiatric practice and research. Psychiatry Res 28(2):193-213

15. Vitiello MV, Larsen LH, Moe KE (2004) Age-related sleep change: gender and estrogen effects on the subjective-objective sleep quality relationships of healthy, noncomplaining older men and women. J Psychosom Res 56(5):503-510

16. Smets EM, Garssen B, Bonke B et al (1995) The Multidimensional Fatigue Inventory (MFI) psychometric qualities of an instrument to assess fatigue. J Psychosom Res 39(3):315-325

17. Brahler E, Gunzelmann T, Hinz A et al (2001) The extent of tiredness and fatigue in the age group over 60 years in a healthy German population sample. Psychotherapeut 46:332-338

18. Allen RP, Picchietti D, Hening WA et al (2003) Restless legs syndrome: diagnostic criteria, special considerations, and epidemiology. A report from the restless legs syndrome diagnosis and epidemiology workshop at the National Institutes of Health. Sleep Med 4(2):101-119

19. Rothdach AJ, Trenkwalder C, Haberstock J et al (2000) Prevalence and risk factors of RLS in an elderly population: the
MEMO study. Memory and Morbidity in Augsburg Elderly. Neurology 54(5):1064-1068

20. Hening WA, Allen RP, Washburn M et al (2008) Validation of the Hopkins telephone diagnostic interview for restless legs syndrome. Sleep Med 9(3):283-289

21. Trenkwalder C, Wetter TC, Stiasny K et al (2001) Restless legs syndrome and periodic limb movements in sleep. Nervenarzt 72(6):425-436

22. Walters AS, LeBrocq C, Dhar A et al (2003) Validation of the International Restless Legs Syndrome Study Group rating scale for restless legs syndrome. Sleep Med 4(2):121-132

23. Ware JE Jr, Sherbourne CD (1992) The MOS 36-item short-form health survey (SF-36). I. Conceptual framework and item selection. Med Care 30(6):473-483

24. Schwarz R, Gunzelmann T, Hinz A et al (2001) Anxiety and depression in the general population over 60 years old. Dtsch Med Wochenschr 126(21):611-615

25. Bloch KE, Schoch OD, Zhang JN et al (1999) German version of the Epworth Sleepiness Scale. Respiration 66(5):440-447

26. Backhaus J, Junghanns K, Broocks A et al (2002) Test-retest reliability and validity of the Pittsburgh Sleep Quality Index in primary insomnia. J Psychosom Res 53(3):737-740

27. Morfeld M, Bullinger M, Nantke J et al (2005) The version 2.0 of the SF-36 Health Survey: results of a population-representative study. Soz Praventivmed 50(5):292-300

28. Zeitlhofer J, Schmeiser-Rieder A, Tribl G et al (2000) Sleep and quality of life in the Austrian population. Acta Neurol Scand 102(4):249-257

29. Schwarz R, Krauss O, Hinz A (2003) Fatigue in the general population. Onkologie 26(2):140-144

30. Rhode AM, Hosing VG, Happe S et al (2007) Comorbidity of migraine and restless legs syndrome-a case-control study. Cephalalgia 27(11):1255-1260

31. http://www.grafstat.de

32. Holzner B, Kemmler G, Meraner V et al (2003) Fatigue in ovarian carcinoma patients: a neglected issue? Cancer 97(6): $1564-1572$

33. Rubinsztein JS, Rubinsztein DC, Goodburn S et al (1998) Apathy and hypersomnia are common features of myotonic dystrophy. J Neurol Neurosurg Psychiatry 64(4):510-515

34. Merkelbach S, Schulz H (2006) What have fatigue and sleepiness in common? J Sleep Res 15(1):105-106

35. Rutkove SB, Matheson JK, Logigian EL (1996) Restless legs syndrome in patients with polyneuropathy. Muscle Nerve 19(5):670-672

36. Krajewski KM, Lewis RA, Fuerst DR et al (2000) Neurological dysfunction and axonal degeneration in Charcot-Marie-Tooth disease type 1A. Brain 123(Pt 7):1516-1527

37. Hening W (2004) The clinical neurophysiology of the restless legs syndrome and periodic limb movements. Part I: diagnosis, assessment, and characterization. Clin Neurophysiol 115(9): 1965-1974

38. Rijsman RM, de Weerd AW (1999) Secondary periodic limb movement disorder and restless legs syndrome. Sleep Med Rev $3(2): 147-158$

39. Aboussouan LS, Lewis RA, Shy ME (2007) Disorders of pulmonary function, sleep, and the upper airway in Charcot-MarieTooth disease. Lung 185(1):1-7

40. Shy ME, Blake J, Krajewski K et al (2005) Reliability and validity of the CMT neuropathy score as a measure of disability. Neurology 64(7):1209-1214

41. Padua L, Shy ME, Aprile I et al (2008) Correlation between clinical/neurophysiological findings and quality of life in Charcot-Marie-Tooth type 1A. J Peripher Nerv Syst 13(1):64-70

42. Vinci P, Serrao M, Millul A et al (2005) Quality of life in patients with Charcot-Marie-Tooth disease. Neurology 65(6):922-924 